\title{
Recent Advances in Optical Molecular Imaging and Sensing
}

\section{Zhen Yuan*}

Bioimaging Core, Faculty of Health Sciences, University of Macau, Macau SAR, China

Optical molecular imaging techniques have experienced tremendous advancement in developing novel imaging and sensing techniques to improve our understanding of cellular and molecular events, which includes the development of microscopic techniques for live cell imaging at super resolution, and macroscopic techniques to monitor molecular events in living organism. The ability to image specific genes, molecular targets and molecular pathways in vivo is having a profound impact in preclinical research designed to develop new animal models of human disease and to evaluate new therapeutic and diagnostic strategies. The very popular optical molecular imaging methods including Bioluminescence Tomography (BLT), Fluorescence Molecular Tomography (FMT), Photoacoustic Tomography (PAT), Optical Coherence Tomography (OCT), Corenkov Luminescence Tomography (CLT), Optical Projection Tomography (OPT), Confocal Laser Scanning Microscopy (CLSM), Selective Plane Illumination Microscopy (SPIM) and Stochastic Optical Reconstruction Microscopy (STORM).

For optical macroscopic imaging of cellular and sub-cellular activity in biological media, optical tomography of bioluminescent and fluorescent reporters in pre-clinical animal models is an important technology for the translational study of disease and drug development. Bioluminescence and fluorescence involve detection of light signatures emitted from living animals at visible or near-infrared wavelengths by a sensitive CCD camera. BLT detects very low levels of light generated due to catalysis of luciferin/coelenterazine substrates by luciferase enzymes and has high sensitivity and low noise. However, more attention has been given to the use of near-infrared (NIR) fluorescent probes in order to achieve high detection sensitivity and to the implementation of FMT to enable accurate and quantitative imaging of protein concentration, function and gene expression in vertebrates in vivo.

Although both BLT and FMT have high throughput rates, they have limited imaging depth $(1 \mathrm{~cm})$, lack of depth resolution, and poor lateral resolution. Importantly, none of the two molecular imaging modalities can visualize agents at both microscopic and macroscopic levels. Recent research interest in laser-induced PAT is rapidly growing, largely because of its unique capability of combining high optical contrast and high ultrasound resolution in a single modality. PAT can visualize the internal three-dimensional (3D) structure and function of soft tissues in multi-scale with high spatial resolution (from 1 micrometer to 1 millimeter in different scales) and excellent optical contrast. It is now becoming one of the most powerful optical molecular imaging methods.

Applications of multimodality molecular imaging that combineFMT, BLT, PET and PAT are growing quickly for recording events from single live cells to whole animals with high sensitivity and accurate quantification. Such approaches have immense potential to track progression of metastasis, immune cell trafficking, stem cell therapy, transgenic animals and even molecular interactions in living subjects, which represent the future and trends of optical molecular imaging technologies.

The topics for optical molecular imaging can be summarized as follows:
1) Optical Methods for 3D in vivo Molecular Imaging

Detection Techniques

NIRS Dyes and Nano probes

FMT

BLT

CLT

X-ray Luminescence Optical Tomography

PAT

2) Multimodal Fusion Techniques for 3D Optical Molecular Imaging in vivo

Fused PAT and FMT

Fused FMT and BLT

Fused FMT and PET

Fused BLT, FMT and CT

Fused FMT, BLT and OCT

Fused PAT and OCT

Fused PAT and ultrasound imaging

Fused FMT, BLT and MRI

*Corresponding author: Zhen Yuan, Bioimaging Core, Faculty of Health Sciences, University of Macau, Macau SAR, China, Tel: 83978445; E-mail: yzhen.star@gmail.com

Received January 29, 2014; Accepted January 30, 2014; Published February 05, 2014

Citation: Yuan Z (2014) Recent Advances in Optical Molecular Imaging and Sensing. J Biosens Bioelectron 5: e128. doi:10.4172/2155-6210.1000e128

Copyright: (c) 2014 Yuan Z. This is an open-access article distributed under the terms of the Creative Commons Attribution License, which permits unrestricted use, distribution, and reproduction in any medium, provided the original author and source are credited. 